\title{
Improving the Efficiency of Gas Turbines During Off-Design Operation by Adjusting the Turbine and Compressor Blade Stagger Angles
}

\section{Schobeiri MT*}

Department of Mechanical Engineering, Texas A\&M University, College Station, Texas, USA

\begin{abstract}
Gas turbines in general and aircraft engines in particular undergo frequently dynamic operations. These operations include the routine start-up, load change and shut downs to cover their operation envelope. The frequency of the dynamic operation depends on the size of the engines and the field of application. Engines for commuter aircrafts and particularly helicopter engines operate more often in an off-design mode compared to large commercial aircraft engines and power generation gas turbines. During these routine operations, the compressor mass flow, the pressure ratio, the combustion chamber fuel and air mass flow as well as turbine mass flow change. These changes affect the engine aerodynamic performance and its efficiency. To avoid the inception of rotating stall and surge, high performance gas turbines are equipped with mechanisms that adjust the stator stagger angles thus aligning the stator exit flow angle to the rotor inlet angle, which reduces an excessive incidence. The reduction of incidence angle not only preserves the stable operation of the compressor, but it also prevents the compressor efficiency from deterioration. The existence of an inherent positive pressure gradient may cause the boundary layer separation on compressor blades leading to the rotating stall and surge. Such condition, however, does not exist in a turbine, and therefore, there has been no compelling reason to apply the blade adjusting method to the turbine component. For the first time, the impact of turbine blade stagger angle adjustment on the gas turbine efficiency during the operation is shown in this paper. Given a statistically distributed load condition, the extensive dynamic simulation reported in this paper shows how the efficiency can be positively affected through proper blade adjustment. For the time dependent operation, the code GETRAN developed by the author was enhanced to include the turbine blade adjustment as a function of time. To conduct the dynamic simulation with turbine stator stagger angle adjustment during a dynamic operation, the full geometry of the Brown Boveri GT-9 gas turbine was utilized. Starting from the reference stagger angle, it is varied within an incidence range of \pm 3 degree. Detailed simulation results show the substantial efficiency improvement through stator stagger blade adjustment.
\end{abstract}

Keywords: Gas turbines; Compressor

\section{Introduction}

Power generation and aircraft gas turbines frequently undergo operation conditions that are different from their design points. While in power generation case the startups, shutdowns and moderate load changes are major short duration off-design operations, the operation of aircraft engines must cover an entire off-design envelope. This is particularly true for engines of small commuter aircrafts as well as helicopters. In the course of these transient operations, the components of the engine are exposed to extreme aerodynamic, thermal, and mechanical stresses that may affect the safety, reliability, and, thus, the operability of the engine. Accurately predicting these stress conditions is crucial in the early stages of the design and development. In this case, engine designer utilizes appropriate material and dimensions to prevent unforseen circumstances.

Operating in a dynamic state, requires randomly changing the fuel mass flow. As a consequence, the turbine power reacts to the change of the fuel flow with a power mismatch between the compressor and the turbine component. As a result, the compressor and turbine mass flow rates change that affect both the compressor and turbine aerodynamics. In the compressor case, the blade boundary layer is exposed to a positive pressure gradient environment. It may cause the boundary layer on compressor blades to separate leading to rotating stall and surge. To avoid the inception of rotating stall and surge, high performance gas turbines are equipped with mechanisms that adjust the stator stagger angles thus aligning the stator exit flow angle to the rotor inlet flow conditions, thus reducing an excessive incidence change. The reduction of incidence angle not only preserves the stable operation of the compressor, but it also prevents the compressor efficiency from deterioration. In turbine case, however, the flow is characterized by a negative pressure gradient environment, which does not cause the blade boundary layer to separate. Accordingly, there has been no compelling reason to apply the blade adjusting method to the turbine component.

Considering the turbine component, the random variation of the turbine mass flow causes the turbine blade incidence angle to deviate from its design point. As a result, the profile, secondary and trailing edge losses will change that affect the thermal efficiency of the engine. To prevent the thermal efficiency from major deterioration, a method of turbine stator stagger angle adjustment is introduced in this paper. It is shown that properly adjusting the stator stagger angle during the engine operation positively impacts the thermal efficiency.

To conduct the current investigations, the full geometry of the Brown Boveri GT-9 gas turbine was utilized. Starting from the reference stagger angle, it is varied within an incidence range of \pm 3 degree. For the time dependent operation, the nonlinear code GETRAN developed by the author was enhanced to include the turbine blade adjustment

*Corresponding author: Schobeiri MT, Department of Mechanical Engineering Texas A\&M University, College Station, Texas 77843-3123, USA, Tel: 778433123; E-mail: tschobeiri@tamu.edu

Received February 09, 2018; Accepted February 19, 2018; Published February 24, 2018

Citation: Schobeiri MT (2018) Improving the Efficiency of Gas Turbines During Off-Design Operation by Adjusting the Turbine and Compressor Blade Stagger Angles. J Appl Mech Eng 7: 302. doi:10.4172/2168-9873.1000302

Copyright: @ 2018 Schobeiri MT. This is an open-access article distributed under the terms of the Creative Commons Attribution License, which permits unrestricted use, distribution, and reproduction in any medium, provided the original author and source are credited. 
as a function of time. Detailed simulation results show the substantial efficiency improvement through stator stagger blade adjustment.

\section{Gas Turbine, Components, System}

The gas turbine GT-9 of Brown Boveri used for this study is a single-spool, single shaft power generation gas turbine. The engine consists mainly of an inlet nozzle, three compressor stage groups, a combustion chamber, a turbine, an exit diffuser, a control system, a generator and several pipes and auxiliary components. The simulation schematic of this engine is presented in Figure 1.

As seen the simulation schematic has a modular structure, where the components are treated as modules, thus any gas turbine can be composed of any number of components. The components are coupled with each other via plena. In this section we discuss only four main components: Plenum, Compressor, Combustion Chamber and Turbine.

\section{Plenum}

Two successive components are connected to each other via a plenum, which is the coupling module between two or more components as shown in Figure 2. The primary function of the plenum is to couple the dynamic information of entering and exiting components. The volume of the plenum is the sum of half of all volumes of the components that enter and exit the plenum. As seen, the inlet components transfer information about the mass flow $\dot{m}_{i}$, the total pressure $P$, the total temperature $T_{0}$, the fuel/air ratio, $f=\dot{m}_{f} / \dot{m}$ air and the water/air ratio $w=\dot{m}_{w} / \dot{m}$ air to the plenum. After entering the plenum, a mixing process takes place, where the afore mentioned

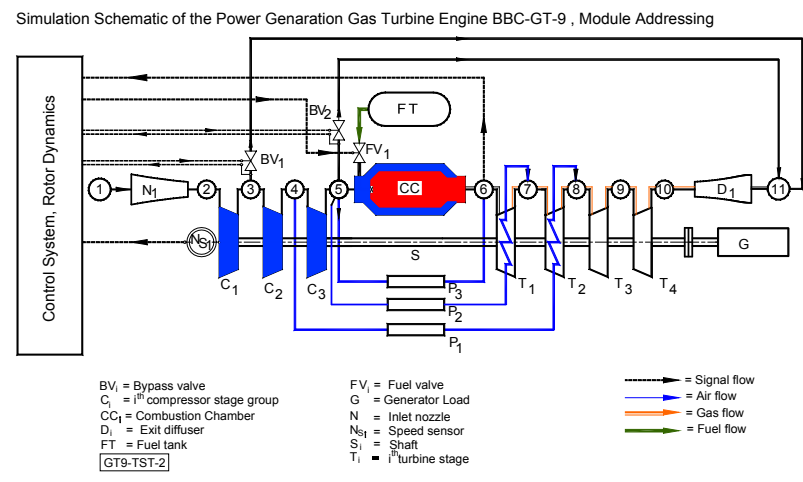

Figure 1: Simulation schematic of BBC-GT9

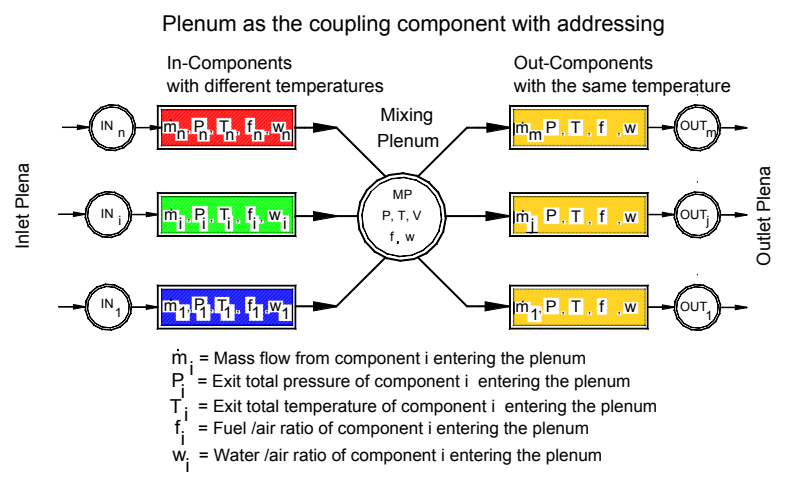

Figure 2: Plenum connecting inlet components to outlet components. quantities reach their equilibrium values. These values are the same for all outlet components exiting the plenum. An example clarifies the role of a plenum: The pipe $\mathrm{P}_{3}$ in Figure 1 connects the plenum 5 with plenum 6. Plenum 5 represents the exit plenum of the HP-compressor. This plenum has certain water content $w$ but not any fuel content $(f=$ $0)$. The exit plenum 6 from the combustion chamber has the same water content $w$ but its fuel air ratio is $f_{\text {cch }} \neq 0$ The combustion chamber mass flow is now mixed with the mass flow through $\mathrm{P}_{3}$ changing its fuel/ air ratio, such that $f_{c c h}>f_{\text {turb }}$. The correct calculation of both fuel/air and water/air ratio is essential for accurate calculation of compressor and turbine power. The plenum is represented by two partial differential equations (PDE) that calculate the pressure and temperature of the plenum. The mass flow calculation, however, occurs in each individual component. Each component represented by its module and its function is described by a number of partial differential equations. Pipes, whose function is to transport mass flow is described by three PDEs, which are equations of continuity, momentum and energy.

The simulation of the dynamic behavior of a recuperator, or a segment of a combustion chamber, however, requires 7 PDEs, 3 PDEs describe the hot side thermo-fluid state, 3 PDEs describe the cold side thermo-fluid state and 1 PDE is the coupling condition between the hot and cold side of the recuperator or combustion chamber segment.

\section{Compressor design and off-design performance}

The prerequisite for simulating the dynamic behavior of the compressor component of a gas turbine is a detailed knowledge of the design point and the blade total loss coefficient at off-design operation. There are three levels of simulation. The first level uses steady state performance maps. It provides global information about the performance behavior of a compressor. It does not contain any detailed information about the individual stage parameters and the stage velocity diagram. However, this information is necessary to construct the compressor performance map. The map is generated either by a stage-by-stage or by a row-by-row compression calculation procedure, which treats the compression process adiabatically, neglecting the heat transfer to/from the blade material. The row-by-row adiabatic compression calculation method is the second level simulation. Compared to the stage-by-stage calculation procedure, the row-by-row method provides a smoother transition from the stator row to rotor row and vice versa with fewer iterations. The third level simulation deals with a diabatic compression process that is necessary for a comprehensive dynamic simulation, where heat transfer from/to the compressor blade material is involved. In calculating the dynamic behavior of the gas turbine, the row-by-row adiabatic calculation method was applied. This calculation delivers the performance map which helps the compressor designer to verify the accuracy of the performance prediction. An example of such calculation is presented in Figures 3 and 4.

Figure 3 exhibits the complete performance map for the lowpressure part of the GT-9 compressor with surge and choke limits. The corresponding efficiency map is shown in Figure 4. Details of generation of compressor performance map using loss coefficient as a function of modified diffusion factor introduced by the author are found in references [1-5].

\section{Combustion chamber}

It generally consists of a primary combustion zone, a secondary zone, and a mixing zone. The primary zone, surrounded by $n$ rows of segments separating the primary combustion zone from the secondary zone and protects the combustion chamber casing from being exposed to high temperature radiation. The actual process of combustion occurs in the primary zone. The mixing in of the secondary air passing 
through holes reduces the gas temperature in the mixing zone to an acceptable level for the gas turbine that follows. The rows of segments in the combustion zone are subjected to a severe thermal loading due to direct flame radiation. Film and convective cooling on both the air and the gas sides cools these segments. The air mass flows required to cool these hot segments flow through finned cooling channels, thereby contributing to the convection cooling of the segments on the air side. The cooling air mass flow exiting from the $j^{\text {th }}$ segment row, affects a film cooling process on the gas side within the boundary layer in the next row of segments. At the end of that process, the cooling air mass flow is mixed with the primary air mass flow, thus, reducing the exit temperature (Figure 5).

Different dynamic simulations can be conducted that reflect the

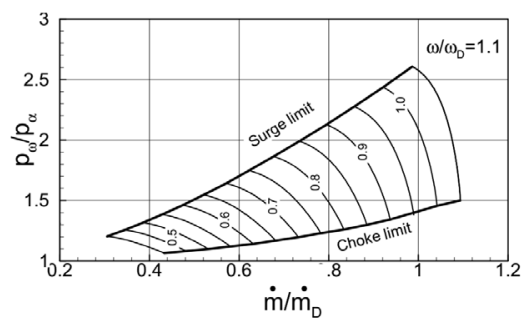

Figure 3: Compressor pressure ratio as a function of relative mas flow with relative angular velocity as parameter.

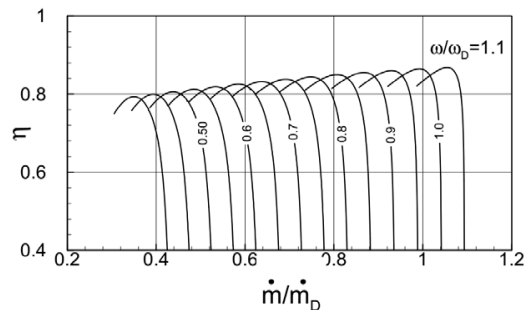

Figure 4: Efficiency as a function of relative mass flow with relative angular velocity as parameter.

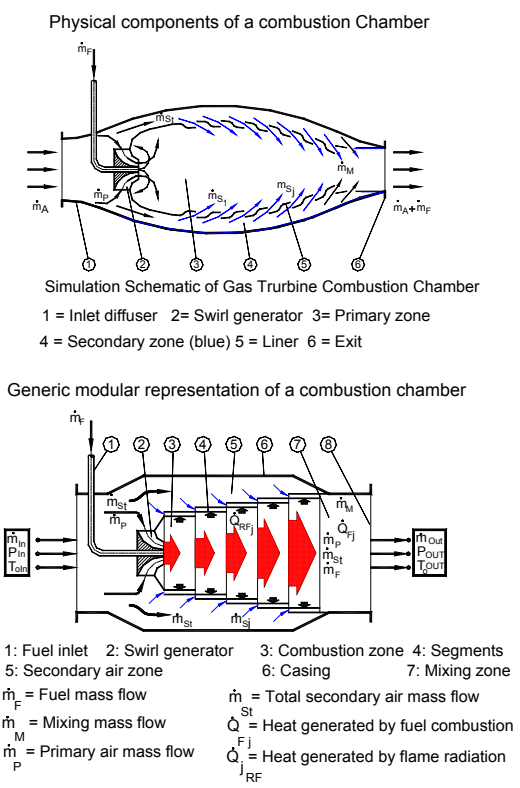

Figure 5: Modular representation of a gas turbine combustion chamber. actual engine test. Startup, shutdown, dynamic load change, rotating stall and surge tests are a few examples. The control system allows the gas turbine to operate in an open and a closed loop. In latter case, the input parameters to the control system are the rotor speed and the turbine inlet temperature with the fuel mass flow, and several valves closing/opening as the output parameters. The dynamic behavior of BBC-GT9 was experimentally determined for routine and adverse transient operations. Its transient data was documented by Schobeiri $[1,2]$.

\section{Turbine design and off-design performance}

The prerequisite for simulating the dynamic behavior of the turbine component is a detailed knowledge of the design point and the blade total loss coefficient at off-design operation.

Changing the turbine blade stagger angle or operating the turbine at an off-design condition causes the flow incidence angle to deviate from its optimum. This deviation in turn affects the individual and the total stage losses and thus the efficiency of the turbine stage. Since an accurate calculation of the individual losses such as profile, trailing edge, secondary flow and ventilation losses is associated with major uncertainties, the author developed a method that directly calculates the stage efficiency at design and adverse off- design conditions. This issue is comprehensively discussed in references [3-5], therefore only a brief description is given below.

Using a row-by-row calculation approach, the method is described briefly in the following. The stator and rotor row efficiencies $\eta^{\prime}$ and $\eta^{\prime \prime}$ at any off-design point are related to their design point efficiencies $\eta^{*}$, and $\eta^{*}$ " through:

$$
\eta^{\prime}=\eta^{*} \cdot\left(1-e^{c^{\prime}}\right), \eta^{\prime \prime}=\eta^{*} "\left(1-e^{c^{\prime \prime}}\right)
$$

where, the exponent $c^{\prime}, c$ ' for the stator, and rotor are: 1

$$
c^{\prime}=A^{\prime}\left[\frac{\alpha_{1}}{\alpha_{1}^{*}}\right]^{B^{\prime}}, c^{\prime \prime}=A^{\prime \prime}\left[\frac{\beta_{2}}{\beta_{2}^{*}}\right]^{B^{\prime \prime}}
$$

and the coefficients and exponents in (2) in are given as:

$$
\begin{aligned}
& A^{\prime}=0.075 \alpha_{2}-7.8460 \\
& A^{\prime \prime}=0.075\left(180-\beta_{3}\right)-7.8460 \\
& B^{\prime}=0.029 \alpha_{2}+0.6107 \\
& \mathrm{~B}^{\prime}=0.029\left(180-\beta_{3}\right)+0.6107
\end{aligned}
$$

All quantities denoted by the symbols $(/, / /)$ refer to stator, rotor respectively. All angles denoted by the symbol $(*)$ refer to design point angles. The coefficients $A^{\prime}, A^{\prime \prime}, B^{\prime}, B$ ', depend on the Renumber, Mach number, and blade geometry. These coefficients were determined for the particular set of turbine blade profiles used in this paper. The angles are measured in degrees and their directions follow the convention in Figure 6. Equations (1) to (4) relate the off-design efficiency of typical turbine blades to the design point efficiency.

With the procedure described above, it is possible to accurately predict the off-design efficiency of a turbine stage provided that the given off-design mass flow permits a normal turbine operation. However, if the mass flow has been reduced to such an extent that the turbine stage is no longer able to produce mechanical energy, the efficiency has to be redefined in such a way that it reflects the dissipative nature of the energy conversion. Example of such an 


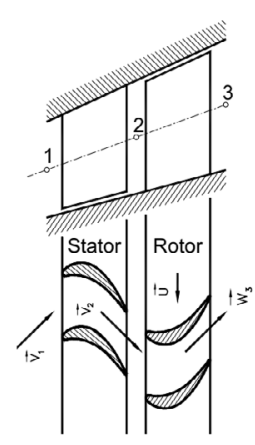

Row arrangement

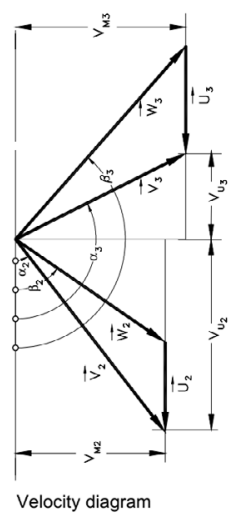

Figure 6: Turbine stage, velocity diagram and angle convention.

operation condition is the shutdown of a steam turbine, where the mass flow is being reduced to zero and the kinetic energy of turbine rotor is dissipated as heat. Detailed treatment of thistreatment of this issue is found in references [3-5].

\section{Dynamic Simulation Code}

For a detailed non-linear dynamic simulation of the gas turbine behavior, the computational platform GETRAN, was used. GETRAN is a generically modular structured computer code for simulating the nonlinear dynamic behavior of single- and multispool high pressure core engines, turbofan engines, and power generation gas turbine engines. The code developed by the author is described in NASA Reports [6-8]. Simulation cases are also found in $[9,10]$. The code GETRAN has been updated and enhanced several times. Its enhancement was reported in the author's textbooks: Turbomachinery Flow Physics and Dynamic Performance $1^{\text {st }}$ and $2^{\text {nd }}$ edition $[3,4]$, and in the very recent text book: Gas Turbine Design, Components and System Integration [11]. In sections that follow, the most enhanced version of GETRAN is used.

\section{Theoretical background}

Dynamic behavior of gas turbine components and systems can generally be described by conservation laws of fluid mechanics and thermodynamics [3-5]. The fluid dynamic process that takes place within a gas turbine system, is of unsteady nature. Considering this fact, the conservation laws of fluid dynamics and thermodynamics must be rearranged such that temporal changes of thermo-fluid dynamic quantities are expressed in terms of spatial changes. A summary of relevant equations is found in [3,4]. For an unsteady flow, the conservation of mass with its derived in reference [3] is:

$$
\begin{aligned}
& \frac{\partial \rho}{\partial t}=-\nabla \cdot(\rho \boldsymbol{V}) \\
& \frac{\partial \rho}{\partial t}=-\frac{\partial}{\partial x_{i}}\left(\rho V_{i}\right) \quad(5)
\end{aligned}
$$

Neglecting the gravitational term, Cauchy equation of motion with its index notation is:

$$
\begin{aligned}
& \frac{\partial(\rho \boldsymbol{V})}{\partial t}+\nabla \cdot(\rho \boldsymbol{V} \boldsymbol{V})=+\nabla \cdot \Pi \\
& \frac{\partial(\rho \boldsymbol{V})}{\partial t}+\nabla \cdot(\rho \boldsymbol{V} \boldsymbol{V})=-\nabla \mathrm{p}+\nabla \cdot \mathbf{T} \quad(6)
\end{aligned}
$$

$$
\frac{\partial\left(\rho V_{i}\right)}{\partial t}=-\frac{\partial}{\partial x_{j}}\left(\rho V_{i} V_{j}\right)-\frac{\partial p}{\partial x_{i}}+\frac{\partial T_{i j}}{\partial x_{j}}
$$

With $\Pi=-\mathrm{Ip}+\mathrm{T}$ as the total stress tensor which is the sum of the pressure tensor and the shear stress tensor, where the unit tensor is defined as $I=e_{i} e_{j} \delta_{i j}$. In addition, the mechanical and the thermal energy equations are used:

$$
\begin{gathered}
\rho \frac{D}{D t}\left(\frac{V^{2}}{2}\right)=-\boldsymbol{V} \cdot \nabla \mathrm{p}+\nabla \cdot(\mathbf{T} \cdot \mathbf{V})-\boldsymbol{T}: \boldsymbol{D} \\
\rho \frac{D u}{D t}=-\nabla \cdot \dot{\boldsymbol{q}}-p \nabla \cdot \boldsymbol{V}+\boldsymbol{T}: \boldsymbol{D}
\end{gathered}
$$

with $T$ as the shear stress tensor and $D$ the deformation tensor. The divergence of the heat flux vector $\nabla . \dot{\boldsymbol{q}}$ represents the amount of heat added to or rejected from the system. Combining both equations in (7) exhibits the total energy balance in terms of internal energy and kinetic energy:

$$
\rho \frac{D}{D t}\left(u+\frac{V^{2}}{2}\right)=-\nabla \cdot \dot{\boldsymbol{q}}+\nabla \cdot(\boldsymbol{\Pi} \cdot \mathbf{v})
$$

Since all components of a gas turbine are considered open systems, it is appropriate to use enthalpy $h$ rather than internal energy $u$. The state properties $h$ and $u$ can be expressed as the sum $h=u+p v$ and the substantial change of the specific static enthalpy is:

$$
\rho \frac{D h}{D t}=-\nabla \cdot \dot{\boldsymbol{q}}+\frac{D p}{D t}+\boldsymbol{T}: \boldsymbol{D}
$$

Replacing the specific internal energy in Eq. (8) by the specific static enthalpy using the relation $u=h-p v$ we arrive at the substantial change of the combined mechanical and thermal energy equation:

$$
\rho \frac{D}{D t}\left(h+\frac{V^{2}}{2}\right)=\frac{\partial p}{\partial t}-\nabla \cdot \dot{\boldsymbol{q}}+\frac{D p}{D t}+\nabla \cdot(\boldsymbol{T} \cdot \boldsymbol{V})
$$

Introducing the total enthalpy, we find its temporal change as derived in [3-5],

$$
\begin{aligned}
& \frac{\partial H}{\partial t}=-k \boldsymbol{V} \cdot \nabla \mathrm{H} \\
& -(\kappa-1)\left(\frac{1}{\rho} \nabla \cdot(\rho \boldsymbol{V})(H+K)+\frac{\boldsymbol{V} \cdot \partial(\rho \boldsymbol{V})}{\rho \partial t}\right)+\left(-\frac{\kappa \nabla \cdot \dot{\boldsymbol{q}}}{\rho}+\frac{\kappa}{\rho} \nabla \cdot(\boldsymbol{V} \cdot \boldsymbol{T})\right)
\end{aligned}
$$

In Eq. (11) the expression $H=h+V^{2} / 2$, is the specific total enthalpy, $K=V^{2} / 2$ the specific kinetic energy and $\dot{q}$ the heat flux vector. Equation (11) can be expressed in terms of total temperature or total pressure. Equations (5) through (11) are written in 3-D form. The index notation of (11) is:

$$
\begin{aligned}
& \frac{\partial H}{\partial t}=-k V_{i} \frac{\partial H}{\partial x_{i}} \\
& -\frac{\kappa-1}{\rho}\left((H+K) \frac{\partial\left(\rho V_{i}\right)}{\partial x_{i}}+\frac{V_{i} \cdot \partial\left(\rho V_{i}\right)}{\partial t}\right)-\frac{\kappa}{\rho}\left(\frac{\partial \dot{q}_{i}}{\partial x_{i}}-\frac{\partial\left(V_{j} T_{i j}\right)}{\partial x_{i}}\right)
\end{aligned}
$$

The index notation of Equations (5) through (12) allows the index variation to cover the range of the indices from 1 to 3 . For a two dimensional time-space calculation, the index $i$ is set $\mathrm{i}=1$. While this is straight forward for continuity and momentum Eqs. (5) and (6), 
the energy equation (12) needs some clarification. In this equation the heat flux vector $\dot{q}$ represents the heat added to or rejected from a component. In case of a turbine or a compressor, while heat is added to or rejected from the blades, the component itself generates/consumes power. In this case, the power generated by the turbine or consumed by the compressor must be integrated into the energy balance. For a two dimensional time-space coordinate Eq. (12) reads:

$$
\begin{aligned}
& \frac{\partial H}{\partial t}=-\kappa_{k} \frac{\dot{m}_{k}}{\rho_{k} S_{k}}\left(\frac{H_{i+1}-H_{i}}{\ddot{\mathrm{A}} x}\right) \\
& -\left(\frac{\kappa-1}{\rho}\right)_{k}\left[\left(\frac{H_{k}+K_{k}}{\ddot{\mathrm{A} x}}\right)\left(\frac{\dot{m}_{i+1}}{S_{i+1}}-\frac{\dot{m}_{i}}{S_{i}}\right)+\frac{\dot{m}_{k}}{\rho_{k} S_{k}^{2}} \frac{\partial \dot{m}_{i+1}}{\partial t}\right] \\
& -\frac{\kappa_{k}}{\rho_{k}}\left(\frac{\ddot{\mathrm{A}} \dot{Q}+\ddot{\mathrm{A}} L}{\ddot{\mathrm{A}} V}\right)
\end{aligned}
$$

with $L=\dot{m} l_{m}$ as the stage power and $l_{m}$ as the specific stage mechanical energy (for detailed derivation [3-5]. The indices $i$ and $i+1$ refer to the inlet and exit of the finite volume under integration and $k$ to their central difference.

It should be noted that the objective of the engine simulation discussed in the following section is to understand the global behavior of its components and the totality of the engine behavior operating under any condition adverse or favorable.

\section{Discussion}

\section{Impact of turbine angle adjustment on gas turbine dynamic performance}

Aero-engines such as those of commuter aircrafts and helicopters are subject to frequent changes of operation conditions. These changes affect the turbine and compressor efficiency and performance. This section deals with a new method to maximize the turbine efficiency operating at frequently changing operation conditions. The change of the operation condition may be periodic, a-periodic or random. In general, any offdesign operation of a gas turbine is associated with changing the mass flow and thus the blade incidence angles of the turbine and components. Incidence variation causes a change of blade losses resulting in lower or higher stage efficiency. Proper adjusting the blade stagger angle to the incidence changes results in lower overall aerodynamics losses, thus an increase of the thermal efficiency of the gas turbine.

\section{Adjusting the turbine blade stator stagger angle}

Before going into the details that follow, Figure 7 shows the impact of the stator stagger adjustment on the stage velocity diagram.

Rotating the stator blade clockwise causes a decrease of the exit flow angle $\alpha_{2}$. As a consequence, the specific turbine stage power will increase causing the thermal efficiency to improve as will be discussed in this section.

Ideally, it would be more effective to adjust both, the stator and the rotor blade stagger angles. However, changing the rotor blade stager angle requires a more complex adjusting mechanism. Furthermore, the high pressure and temperature operating environment adds to the inherent mechanical complexity. Thus, the adjustment of rotor blade stagger angle is not practical to be implemented, therefore, we consider adjusting the stator stagger angle only. The turbine stage with the velocity diagram with and without adjusting the stator is shown in Figure 7 , with the negative and positive changes of the stagger angle

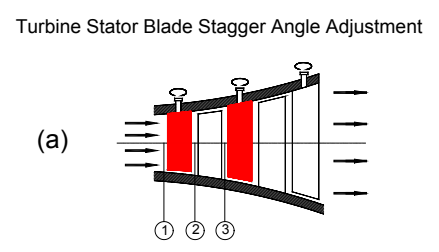

(b)
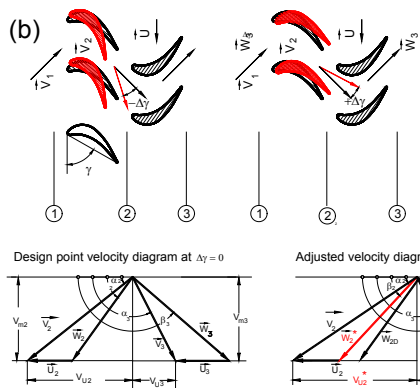

(c)

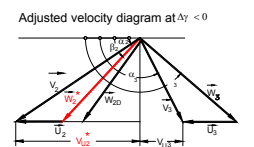

(d)

Figure 7: (a) Turbine component with stator blade adjustment, (b) Adjusted stator blades, (c) Design velocity diagram, (d) Adjusted velocity diagram.
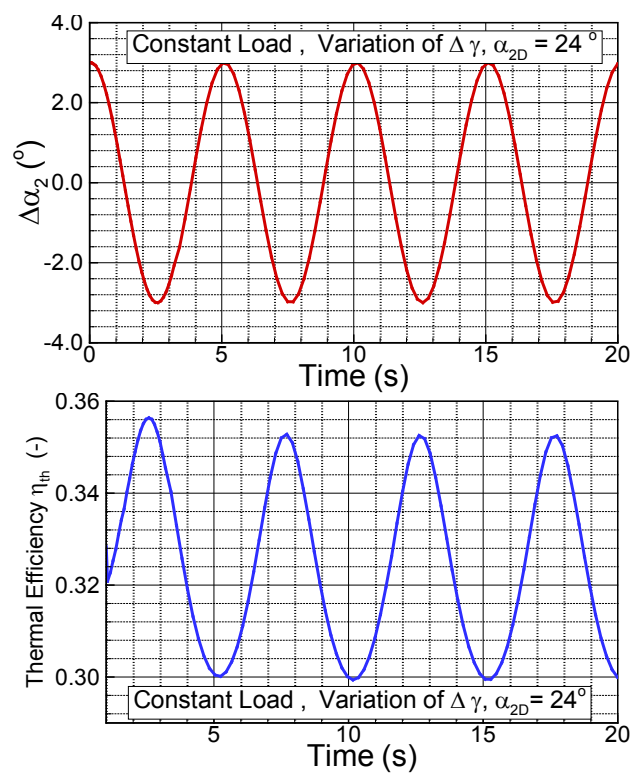

Figure 8: (Top) Periodic variation of the stagger angle as a function of time, (bottom) variation of thermal efficiency as a function of time due to changes of exit flow angle.

$\Delta \gamma(\mathrm{a}, \mathrm{b})$. As seen a negative $\Delta \gamma$ in Figure 7(d) causes an increase in specific stage power represented by $v_{u 2}^{*}+V_{u 3}$ compared with the one of the design $\Delta \gamma=0$ shown in Figure 7(c). Similarly, a positive $\Delta \gamma$ results in a decrease of the specific stage power [12].

\section{Change of stagger angle at constant power schedule}

To demonstrate the effect of the stator angle, change on the efficiency of a gas turbine, we first assume a constant power schedule and change the first stator stagger angle $\Delta \gamma$ periodically from $\Delta \gamma=-3^{\circ}$ to $\Delta \gamma=+3^{\circ}$ while the engine is running, Figure 8 . The $\Delta \gamma$-variation causes a corresponding variation of the exit flow angle $\alpha_{2}$. As the stator exit flow angle reduces, the stator flow deflection from inlet to exit increases leading to an increase in stage specific power and thus a greater thermal efficiency as shown in Figure 7 (d). The thermal efficiency changes 
periodically and reaches its highest value at lowest negative $\Delta \alpha_{2}$, (Figure 8). Figure 9 shows a diagram that reflects the contents of both diagrams in Figure 8 (top and bottom) namely $\eta_{\text {th }}=f(\mathrm{t})$ and $\Delta \alpha_{2}=f(\mathrm{t})$, from which the thermal efficiency $\eta_{\text {th }}=\mathrm{f}\left(\Delta \alpha_{2}\right)$ is constructed.

The thermal efficiency plotted in Figure 9 has an upper and a lower branch. At each $\Delta \alpha_{2}$, there are two efficiency values. The upper branch represents higher efficiency than the lower one whenever $\Delta \alpha_{2}$ has the tendency to assume negative values.

This is clearly explained as one starts from $\Delta \alpha_{2}=0$. Whenever, $\Delta \alpha_{2}$ moves in negative direction, $\eta_{t h}$ increases and vice versa. Passing through several periods of time, the thermal efficiency $\eta_{\text {th }}$ assumes values between 0.3 to 0.35 .

Considering the design point thermal efficiency of $\eta_{\text {th }} \approx 32 \%$, at a stator exit flow angle of $\alpha_{2}=24^{\circ}$ with $\Delta \alpha_{2}=0$, the angle adjustment has brought an increase of almost $3.5 \%$. Given the fact that even an increase of $0.5 \%$ is relevant for turbine efficiency evaluation, an increase of $3.5 \%$ is considered substantial. As Figure 9 shows, for the region where, $\Delta \alpha_{2}>0$ the efficiency substantially decreases below its design point. As a result, the engine control system will have to be programmed such that this region is avoided, when adjusting the stagger angle.

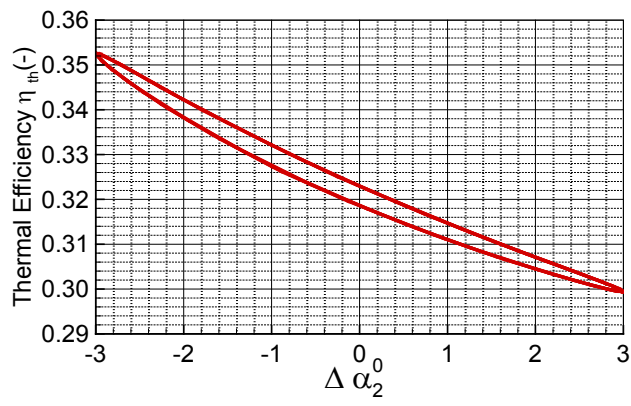

Figure 9: Thermal efficiency as a function of $\Delta \alpha_{2}=0$.
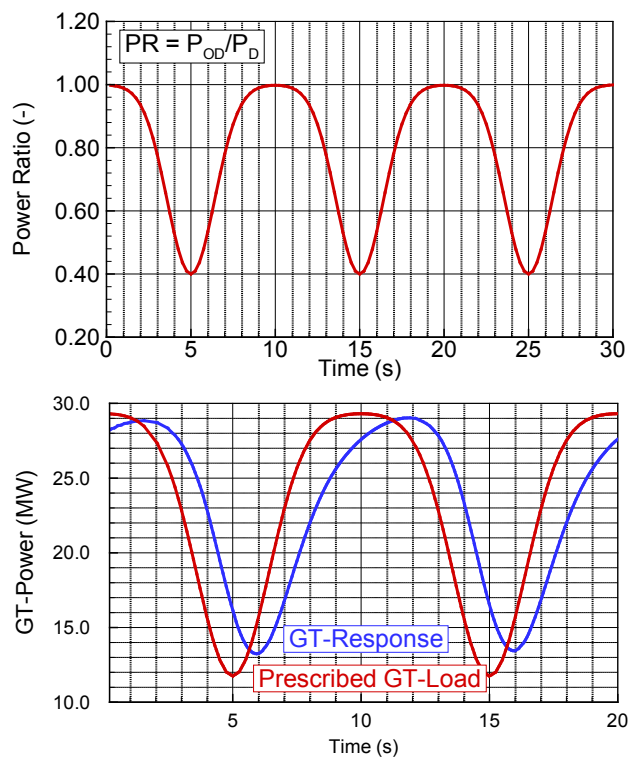

Figure10: (Top) Power ratio given by the operator, (bottom) the prescribed turbine power and the gas turbine response.

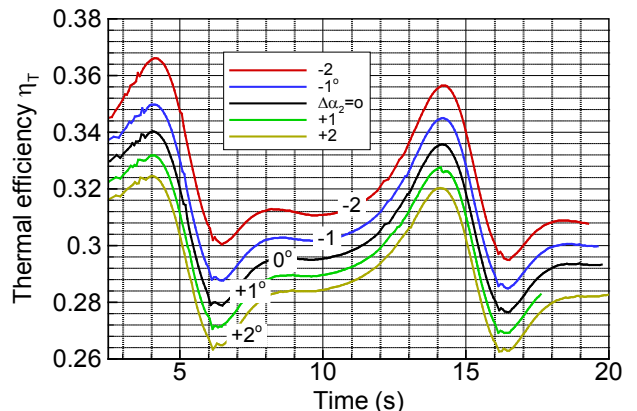

Figure 11: Thermal efficiency as a function of time with different $\Delta \alpha_{2}$ as parameter and Gaussian load distribution.

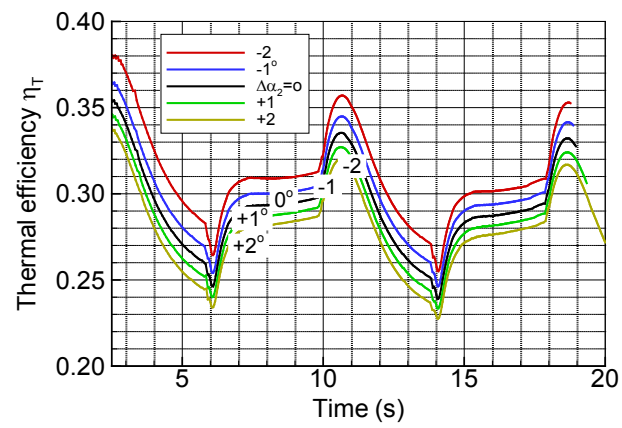

Figure 12: Thermal efficiency as a function of time with different $\Delta \alpha_{2}$ parameter and a step function as the load distribution.

\section{Variable turbine load schedule at constant stagger angle}

We now consider a load schedule that is changing with time. Taking into account the random nature of the events that require changing the stagger angle, we apply for the gas turbine a Gaussian load distribution as a function of time. Figure 10 (top) exhibits the power ratio PR, which reflects the load schedule prescribed by the test bed operator. It is the ratio of the actual off-design power distribution $P_{O D}$ and the design power $P_{D}$. For $P R=1$, the gas turbine runs at its $100 \%$ rated output, whereas for $P R=0.4$ it delivers only $40 \%$ of its design power. The power ratio distribution is presented in Figure 10, whereas the Figure 10 shows the actual power output and the gas turbine response to the schedule.

As seen, there is a time lag between the prescribed load and the engine response. This lag represents the controller characteristic and the reaction of the gas turbine system as a whole.

Using the scheduled power ratio PR from Figure 10 (top), the dynamic simulation of the gas turbine BBC-GT-9 is conducted and the efficiency diagram is presented in Figure 11. For each simulation, the stator $\Delta \alpha_{2}$ was kept constant. Figure 11 exhibits a composite picture of the thermal efficiency envelope as a function of time for five different stagger angle differences $\Delta \alpha_{2}$ including $\Delta \alpha_{2}=0$ which corresponds to the design point. This figure shows three transient phenomena:

(1) The overall efficiency has increased with negatively increasing $\Delta \alpha_{2}$.

(2) At $\Delta \alpha_{2}=-2^{\circ}$ the efficiency distribution has shifted up close to $1.5 \%$ point.

(3) The efficiency distribution region between the two efficiency peaks, which is occupied by the off-design, has experienced a significant increase.

Thus, tuning the stagger angle to the power schedule exhibits a 
practical solution to prevent the efficiency deterioration during adverse off-design operation. This method can be applied to aircraft engines as well as to power generation ones that are exposed to frequent load changes. In the above investigation, a Gaussian distribution was presented as one of the possible power schedules to reduce the efficiency deterioration during the dynamic engine operation. The results are presented in Figure 11. Instead of Gaussian distribution, any other load schedule can be used. As an alternative, a step function is given that provides similar composite picture given in Figure 12.

As seen in both Figures 11 and 12, there is a close interaction between the load schedule and stagger angle variation. Optimizing this interaction with thermal efficiency as a target function is the task of the engine control system. The control system can provide its optimized power schedule that accounts for the stagger angle change.

\section{Performance of Gas Turbine Components}

\section{Control system}

Starting with the control system, Figure 12 shows a rapid increase of the relative shaft speed as a result of power schedule. This triggers the control system to react correspondingly and quickly closing the fuel valve to reach the minimum fuel mass flow at $\dot{m}_{F}=1.429 \mathrm{~kg} / \mathrm{s}$. During the closing of fuel valve, the turbine power reduces while the

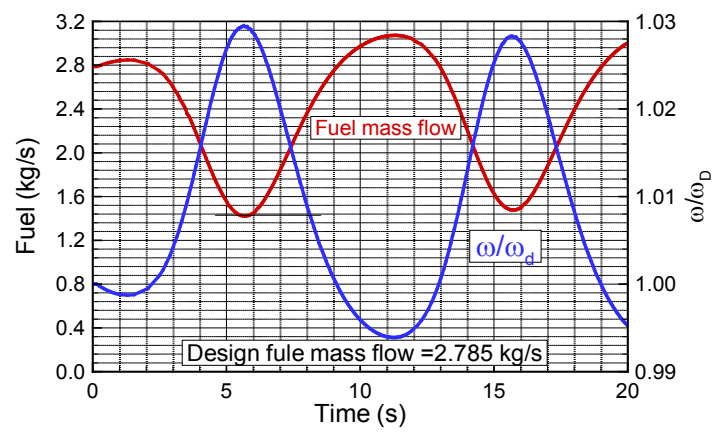

Figure 13: Control system action.
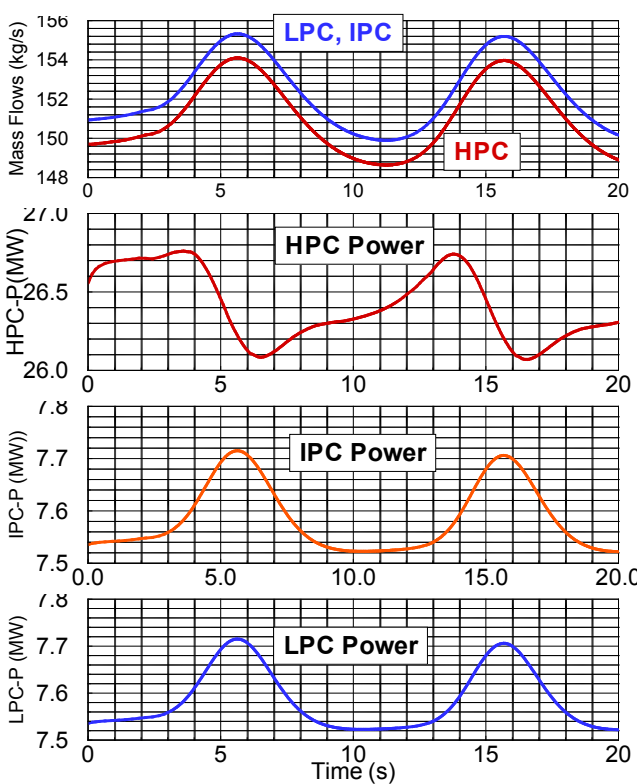

Figure 14: Case 1: Compressor mass flow and power at constant stagger angle but variable load schedule.
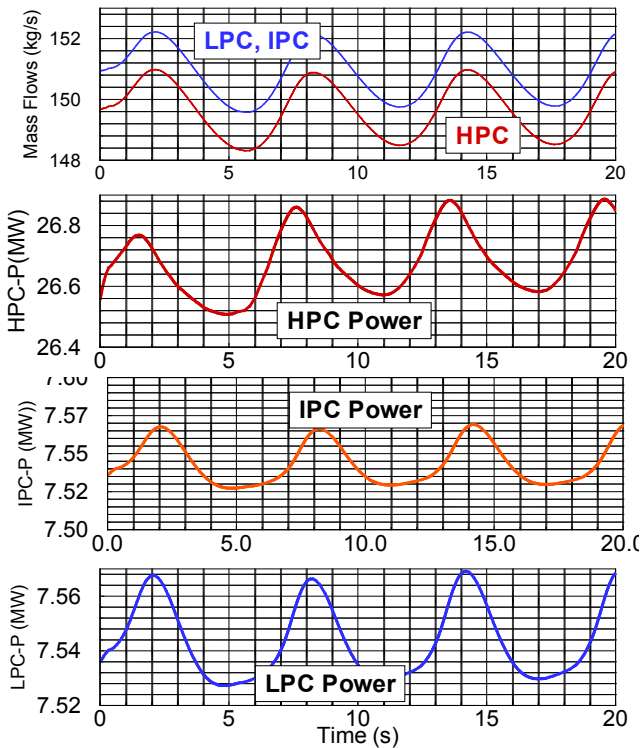

Figure 15: Compressor mass flow and power for a constant load schedule and variable stagger angle as a function of time.

rotor continues to accelerate until it has reached its maximum speed. Once the maximum rotor speed is reached, its slope becomes negative causing the fuel valve to open and the fuel mass flow increases. The same procedure repeats itself as a function of the stagger angle variation (Figure 13).

\section{Compressor components}

To establish the compressor performance behavior under two distinctly different operating conditions, two cases were investigated. In case 1, shown in Figure 14, the load schedule was varied at a constant stagger angle. As seen, the mass flow through the low-pressure compressor (LPC) and the intermediate pressure compressor (IPC) is different from the mass flow through the high pressure compressor (HPC). The difference in mass flows is transferred from plenum 4 through pipe P1, Figure 1, for cooling purposes.

In contrast to Case 1, the stagger angle at constant load schedule is varied in case 2 . These variations directly affect the efficiency and performance of the LP-, IP- and HP-compressor parts as seen in Figures 14 and 15. In Case 1, Figure 14, the load schedule was varied using the Gaussian distribution. The stagger angle variation in case 2 uses a sine function for changes of stagger angle.

\section{Turbine component}

The variations described above not only affect the compressor performance, but it also impacts the performance of the combustion chamber and turbine. For the turbine component, the mass flow, inlet and exit temperatures as well as the turbine power is plotted in Figures 15 and 16 . For varying and constant power distribution. As seen these, properties follow the Gaussian load schedule. The turbine mass flow follows the course of the combustion chamber mass flow, where the fuel is added to the mas flow exiting HPC-part. Also, the inlet and exit temperature follow the load schedule (Figure 17).

\section{Adjusting Compressor Stator Blades}

For the sake of completeness, in the following, the stagger angle adjustment method presented above is applied to the compressor component of a gas turbine. The stagger angle adjustment prevents the 
inception of compressor instability that may lead to rotating stall and surge. To demonstrate the surge protective capability of this method, the gas turbine GT-9 utilized in Section 2 had to be redesigned (Figure 18).

Instead of having four turbine stages that cover the compressor power and delivers net power, we allocate three stages to drive the compressor and one power turbine stage that generates the net power. Thus, we have a gas generator and an independent power turbine that is connected to the gas generator aerodynamically. The purpose of this redesign was to be able to force the gas turbine into rotating stall and surge, since GT-9 in its original configuration is very surge resistant. As seen in Figure 18, the rotational speed controller is connected to the power shaft, thus sensing the rotational speed of the power shaft and its time derivative to control the fuel mass flow.

\section{Case I: Simulation of compressor surge}

Starting from a steady state operating point, the dynamic behavior of the above engine is simulated for a transient operation which is controlled by a prescribed load schedule that acts on the power shaft as
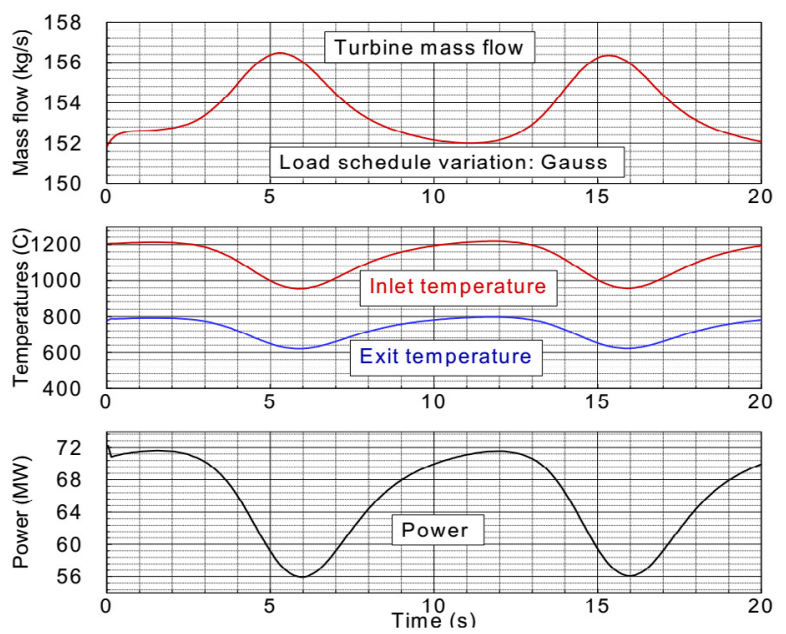

Figure 16: Turbine mass flow, temperature and power as a function of time for Gaussian distribution.
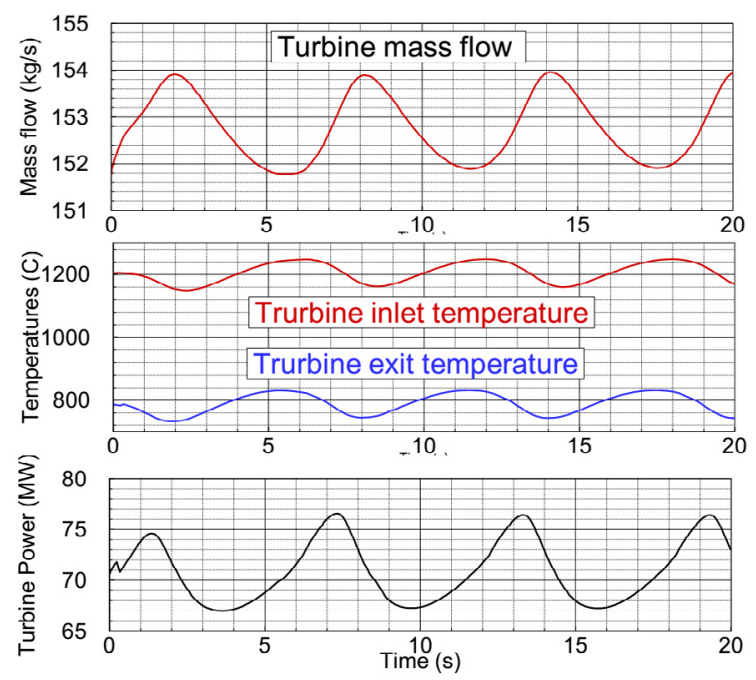

Figure 17: Turbine performance at variable stagger angle and constant load.

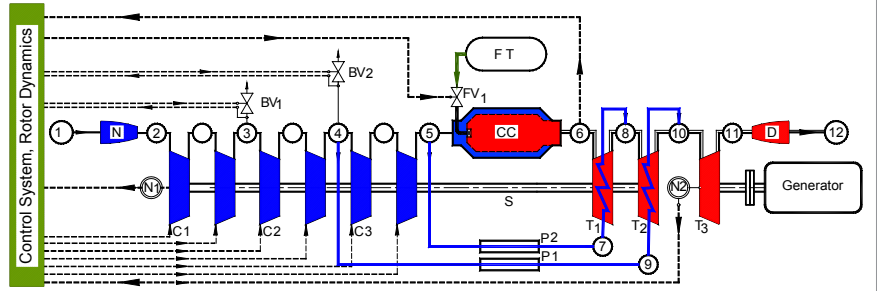

GT1S2S

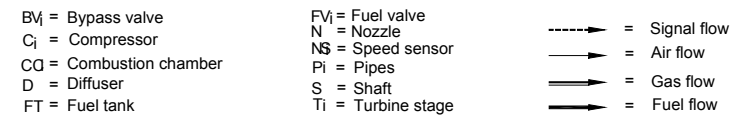

Figure 18: GT-9 with split shaft.
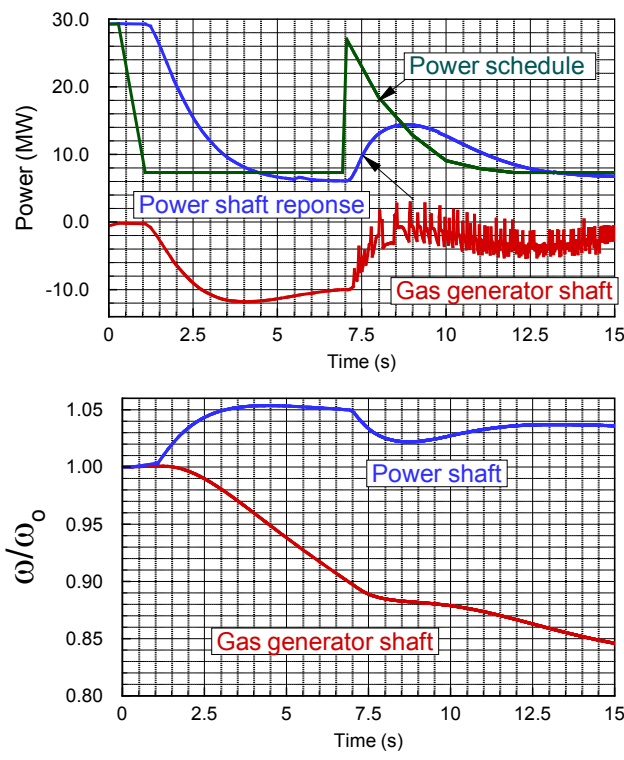

Figure 19: Turbine power and rotational speed.

shown in Figure 19 (top). Following this schedule, we first simulate an abrupt loss of load that represents a partial generator trip. Because of the sudden loss of load, the power generation shaft reacts to this event with a corresponding increase in rotational speed, Figure 19 (bottom). This increase in rotational speed causes the controller to trigger a rapid throttling of the fuel mass flow. The throttling process lasts until a constant idling speed of the power shaft is attained, Figure 19. After about seven seconds, full load is suddenly added and then reduced slowly such that after the completion of load addition, the gas turbine is supplying $25 \%$ of its rated load, Figure 19 (top). The rotor reacts to this sudden addition of load with a sharp decrease in rotational speed. That, in turn, causes a quick opening of the fuel valve. During this process, the power generation capability of the gas generation turbine deteriorates significantly causing a major power imbalance between the turbine and compressor components. This imbalance results in a continuous decrease of the compressor rotor speed, Figure 19 (bottom), causing the compressor to operate partially in rotating stall and surge regimes. As shown in Figure 20, reducing the rotor speed below $90 \%$ forces the LP- compressor stage group into rotating stall and a short duration surge with a reversal in the mass flow direction, Figure 20. Since the magnitude of the reversed mass flow is relatively small and of short duration, a total engine mass flow reversal does not occur. 
The IP-compressor stage group exhibits similar instability behavior as shown in Figure 20, where the compressor mass flow reversal occurs at slightly lower frequency and almost the same amplitude. The HP-stage group displays a distinctively different behavior as shown in Figure 20. While the mass flow experiences a fluctuation at a similar frequency, the amplitude remains always positive. These fluctuations apparently are not caused by the HP-stage group itself and are propagated downstream from LP- and IP-parts respectively. This behavior is fully consistent with the continuity requirement that leads to an integrally positive mass flow rate because of the high frequency and short duration mass flow reversal in LP- and IP-compressors.
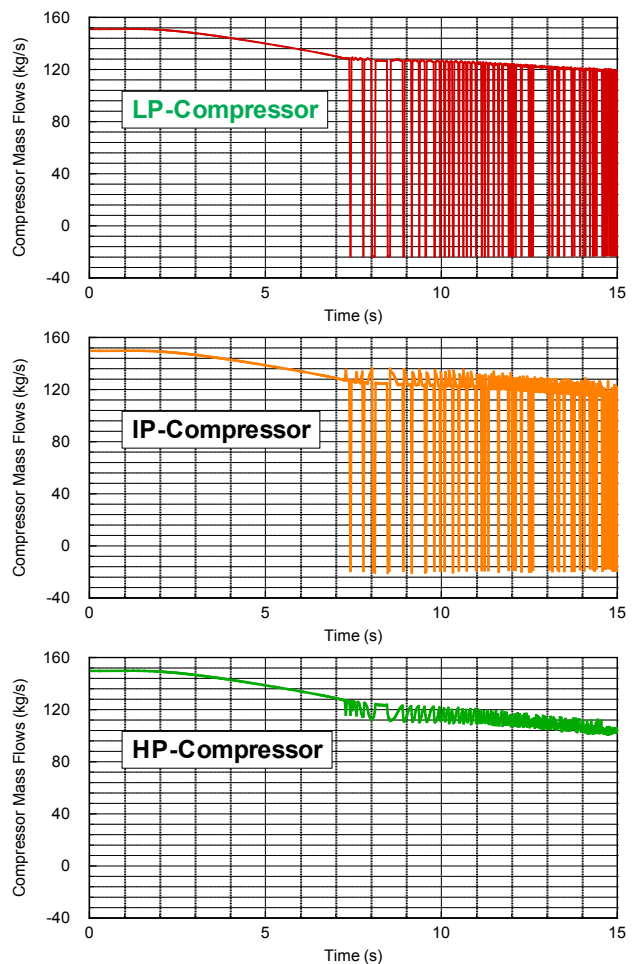

Figure 20: Unstable behaviour of LPC and IPC.

Compessor Stator Blade Stagger Angle Adjustment Design operation: $\gamma$

$\gamma=$ function of time
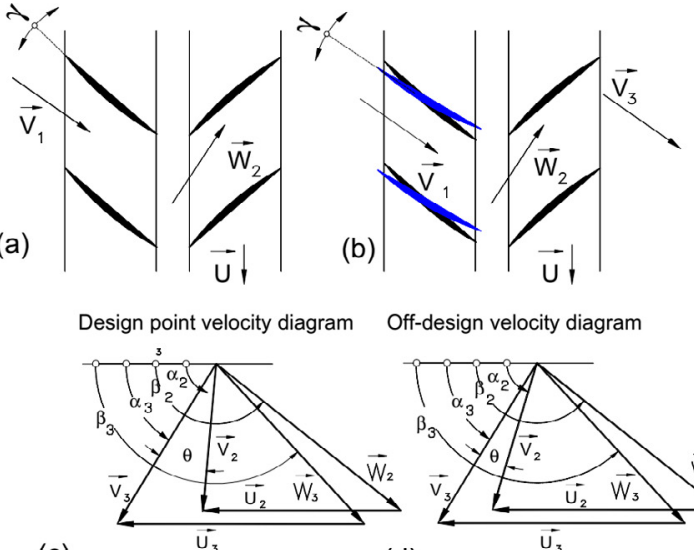

Design point velocity diagram

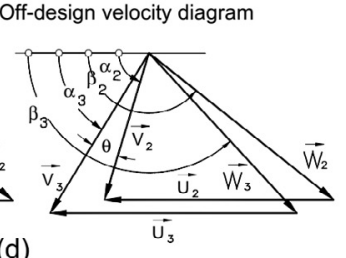

(d)

Figure 21: Stator blade adjustment
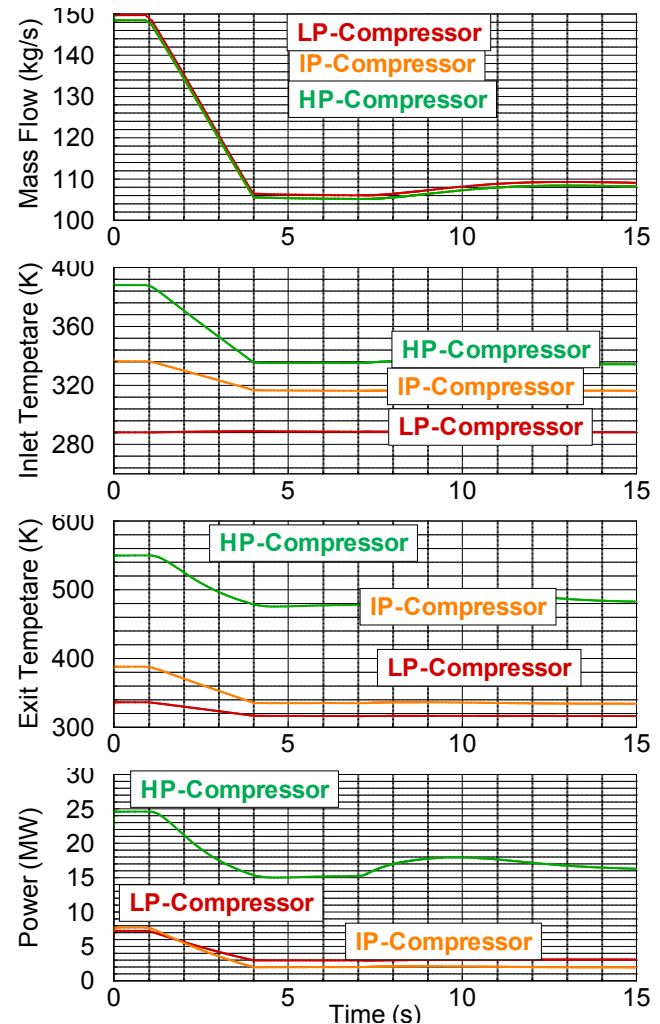

Figure 22: Performance behaviour of compressor component during the stator blades adjustment.

\section{Case II: Active instability and surge prevention by stator stag- ger angle adjustment}

The instability and surge prevention and aerodynamic control discussed in this paper is applied to the gas turbine engines with adjustable compressor stator blade rows. It is the most effective active aerodynamic control mechanism. In contrast to the countless active control procedures found in open literature, this type of control procedure is held as proprietary by the engine manufacturer. Consequently, there is little information in open literature. Figure 21 shows schematically the configuration of a compressor stage with adjustable stator blade rows. As Figure 21(a) shows the stator blade with fixed stagger angle, whereas Figure 21(b) can be turned circumferentially by an electric motor. Pressure sensors downstream of each row communicate the pressure condition to the engine main controller, which decides in which direction the stagger angle $\gamma$ st should be changed. Figures 21(c) and 21(d) show schematically the effect of blade adjustment on the stage velocity diagram. Figure 21(c) exhibits the stator row in its design stagger position under an adverse operation condition which is associated with an increase in compressor pressure ratio. This pressure ratio, however, is established by a deflection angle $\theta$ that may cause a boundary layer separation on stator and rotor blades thus, leading to an inception of rotating stall and surge. To prevent this, the stagger angle $\gamma$ is adjusted resulting in a reduced $\theta$, Figure 21(d). The flow deflection along the stator or rotor cascade with the velocity diagram is shown in Figure 21(c).

To prevent the compressor instability and surge, the stagger angles of the LP, and IP-compressor stage groups were dynamically adjusted. Similar to Case I, the engine was forced into an adverse operation condition with the same load schedule as shown in Figure 19. 

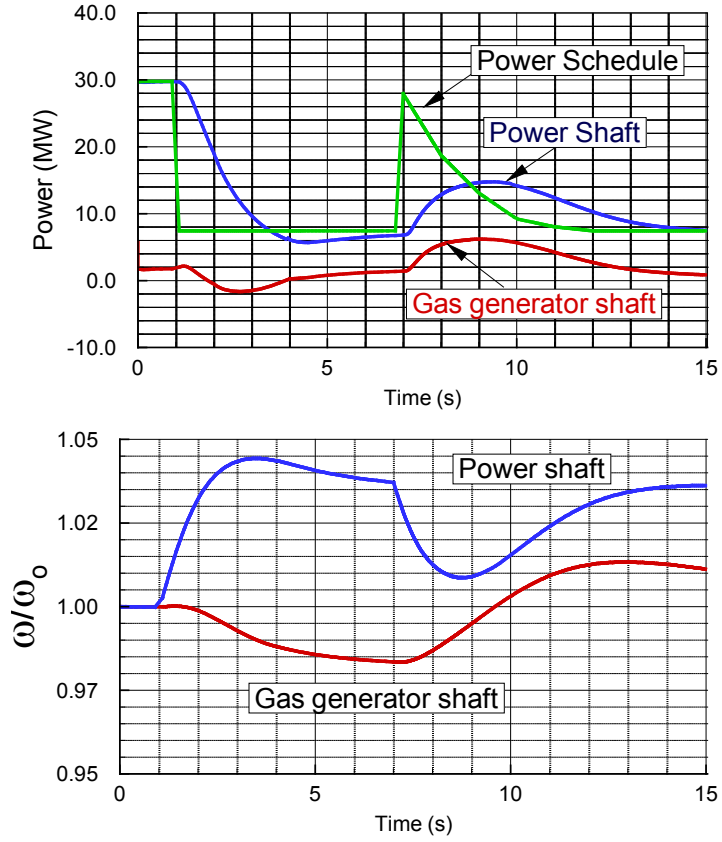

Figure 23: Power and rotational speed behaviour of the gas turbine after compressor stagger adjustment.

Starting from the steady state point, in accordance with the load schedule displayed in Figure 19, first a generator loss of load was simulated. The power generation shaft responds to this event with a rapid increase in rotational speed, which triggers closing of the fuel valve. As a consequence, without active control, the power of the gas generation turbine would not be sufficient to cover the power consumption of the compressor. As observed in Case I this imbalance of power has led to a decrease in rotational speed of the gas generation shaft, which forced the first two compressor stage groups into an unstable regime.

In order to avoid the power imbalance of the gas generation shaft, the stagger angle of the stator blades of the LPC- and IPC stage groups are continuously reduced according to the prescribed schedule plotted in Figure 22. This procedure starts immediately at the time, where loss of load occurs and lasts until the prescribed $\gamma$ - values at $t=4 \mathrm{~s}$ are reached. These values are kept constant for the rest of the simulation. As shown, it is sufficient to reduce the stagger angle of the LP and IP compressors, while the stagger angle of the HP-compressor remained unchanged. This intervention causes a substantial shift in the surge limit preventing all three compressor stage groups from entering into the instability regime. This is shown in Figure 22, where the compressor mass flow does not experience any fluctuations caused by instability.

The stable operation of the compressors is reflected in Figure 23, where the load schedule, Figure 23, and the response of power generator and gas generator turbines are under an adverse transient operation condition. In contrast to the unadjusted Case I shown, no power fluctuateons are encountered.

The rotational speed behavior of the gas generator shaft shown in Figure 23 is substantially different from the one shown in case I.
The positive power difference of the gas generator shaft prevents its rotational speed from decreasing. This moves the compressor to a more stable operation regime. The power generator shaft behaves similar to the one in case I, however it does not reveal any fluctuation.

\section{Conclusion}

The impact of the turbine and compressor blade stagger angle adjustment on the efficiency and performance of gas turbines during a dynamic operation was investigated. The adjustment procedure was applied to the first stator of the turbine component of the gas turbine BBC-GT-9. Two different dynamic scenarios were simulated. In the first simulation, the stagger angle was kept constant whereas the load schedule was varied following a Gaussian distribution. In the second simulation, the load schedule was kept constant and the stagger angle varied as $\Delta \gamma= \pm 3^{\circ}$. It was shown that with a negative $\Delta \gamma$, the thermal efficiency can improve more than one percentage point. Similar increase was observed using proper load schedule.

To complete the investigations, the procedure was applied to the compressor component of the gas turbine GT-9. It was shown that the adjustment procedure can prevent the inception of instability. Adjusting the LP- and IP-compressor stagger angles caused a significant shift in surge limit and established a fully stable operation regime for all three compressor parts. Since all components of a gas turbine function as an integrated system, adjusting the turbine or compressor blade stagger angles cannot be viewed as isolated events. A multi-variable control system has to coordinate their interaction and that is the task for future work.

\section{References}

1. Schobeiri T (1985) COTRAN, The computer code for simulation of unsteady behavior of gas turbines.

2. Schobeiri $T$ (1985) Digital computer simulation of the dynamic response of gas turbines. VDI-Annual J Turbomachinery: 381-400.

3. Schobeiri M (2005) Turbo-machinery flow physics and dynamic performance.

4. Schobeiri M (2017) Gas turbine design, components, system and off-design operation. New York, USA.

5. Schobeiri MT (1995) Final report on GETRAN: The generic, modularly structured computer code for simulation of the transient behavior of single- and multi-spool aero and power generation gas turbine engines.

6. Schobeiri MT, Attia M, Lippke C (1994) Nonlinear dynamic simulation of single and multi-spool core engines, part 1: theoretical method. AIAA J Propulsion and Power 10: 855- 862

7. Schobeiri MT, Attia M, Lippke C (1994) Non-linear dynamic simulation of single and multi-spool core engines, part 2: modeling and simulation cases. AIAA J Propulsion and Power 10: 863-867.

8. Schobeiri MT (1997) Advanced compressor loss correlations, Part 2 Experimental verifications. Int J Rotating Machinery 3:179-187.

9. Schobeiri MT (1998) A new shock loss model for transonic and supersonic axia compressors with curved blades. AIAA, J Propulsion and Power 14: 470-478.

10. Schobeiri MT (1997) Advanced compressor loss correlations, part 1: Theoretical aspects. Int J Rotating Machinery 3:163- 177.

11. Schobeiri T (1986) A general computational method for simulation and prediction of transient behavior of gas turbines. ASME.

12. Schobeiri MT, Abouelkheir M, Lippke C (1994) GETRAN: A generic, modularly structured computer code for simulation of dynamic behavior of aero-and power generation gas turbine engines. ASME Transactions, J Gas Turbine and Power 1: 483-494. 